\title{
Topology Preserving Discretization Schemes for Digital Image Segmentation and Digital Models of the Plane
}

\author{
Alexander V. Evako \\ Npk Novotek, Laboratory of Digital Technologies, Moscow, Russia \\ Email: evakoa@mail.ru \\ Received 30 May 2014; revised 30 June 2014; accepted 9 July 2014 \\ Copyright (C) 2014 by author and OALib. \\ This work is licensed under the Creative Commons Attribution International License (CC BY). \\ http://creativecommons.org/licenses/by/4.0/

(c) (i) Open Access

\begin{abstract}
In this paper, discretization schemes are defined and studied that allow us to build digital models of 2-dimensional continuous objects with the same topological properties as their continuous counterparts. In particular, it is shown that the digital model of the plane is necessarily the digital 2-surface defined and studied in the context of digital topology. It is shown that in proposed discretization schemes, any required resolution can be used in any area of the object of interest. Finally, we describe a method, which transforms regions of interest produced by the image acquisition process into digital spaces with topological features of the regions.
\end{abstract}

\section{Keywords}

Digital Image, Discretization, Digitization, Cover, Plane, Digital Model

Subject Areas: Computer Vision, Computer Graphics and Visualization, Image Processing, Radiology \& Medical Imaging

\section{Introduction}

Digital topology studies geometrical and topological properties of digital images arising in many areas of science including fluid dynamics, geoscience, neuroscience and medical imaging. Integrating topological features into discretization schemes in order to generate topologically correct digital models of anatomical structures is critical for many clinical and research applications, where particular regions of the object require a fine resolution while a relatively coarse resolution can be used over the rest of the object of interest (see [1] [2]).

In the present paper, discretization schemes preserving topology of the object of interest are introduced. These schemes let obtain more detailed geometrical and topological information about the specific regions of the object. 
The shape and the size of individual elements can be arbitrary within the framework of the proposed scheme that allows representing objects with fine anatomical details. Another feature is that the digital model of a 2-dimensional continuous object is necessarily a digital 2-surface preserving the topology of the object. The approach studied in this paper is based on locally centered lump (LCL) discretization of n-dimensional objects.

In recent years, there has been a considerable amount of works devoted to building two-, three- and n-dimensional discretization schemes and digital images (see, e.g., [3]-[12]).

The material to be presented below begins with basic definitions and results related to discretization schemes for continuous objects in Section 2. We study properties of LCL covers of 1- and 2-dimensional continuous objects and formulate conditions for a cover of the plane to be the LCL cover. It is shown that one can choose an LCL cover with a required resolution in any region of interest, which is extremely important in medicine. In Section 3, we prove that for any LCL cover of the plane, the digital model is necessarily a digital 2-surface. A trivial result of this consideration is that there are infinitely many non-isomorphic digital models of the plane. A method is described that constructs digital models of areas of interest with any required resolution.

\section{LCL Collections of 2-Cells and LCL Covers of Continuous Objects}

In this section, we use intrinsic topology of an object, without reference to an embedding space.

We say that a set $D$ is a 2-cell if it is homeomorphic to a closed unit square, a set $D$ is a 1-cell or a segment if it is homeomorphic to a closed unit segment, a set $C$ is a circle or a 1-sphere if it is homeomorphic to a unit circle. We denote the interior and the boundary of an n-cell $D, n=1$, 2, by $\operatorname{Int} D$ and $\partial D$ respectively. Note that $D=D \bigcup \operatorname{Int} D$. The boundary of a 1-cell is two endpoints, the boundary of a 2-cell is a circle. The 0-cell $D$ is a single point for which $\partial D=\varnothing$. Facts about n-cells that we will need in this paper are stated below.

\section{Facts 2.1}

- If $C$ is a circle and $D$ is a 1-cell contained in $C$ then $C$-Int $D$ is a 1-cell.

- Let $C_{1}$ and $C_{2}$ be 1-cells such that $C_{1} \cap C_{2}=\partial C_{1} \cap \partial C_{2}=v$ is an endpoint of $C_{1}$ and $C_{2}$. Then $C_{1} \cup C_{2}=E$ is a 1 -cell.

- Let $D_{1}$ and $D_{2}$ be 2-cells such that $D_{1} \cap D_{2}=\partial D_{1} \cap \partial D_{2}=D^{1}$ is a 1- cell. Then $D_{1} \cup D_{2}=B$ is a 2-cell. Definition 2.1

Let $W=\left\{X_{1}, \cdots, X_{s}\right\}$ be a collection of sets. $W$ is called a locally centered collection (LC collection) if for all $m, k \in Q \subseteq\{1, \cdots, s\}$, from condition $X_{k} \cap X_{m} \neq \varnothing, m \neq k$, it follows that $\bigcap\left\{X_{k}: k \in Q\right\} \neq \varnothing$.

\section{Definition 2.2}

1) Let $W=\left\{C_{1}, C_{2}, \cdots\right\}$ be a collection of 1-cells. $W$ is called a locally lump collection (LL collection) if:

a) From condition $C_{k} \cap C_{m} \neq \varnothing, m \neq k$, it follows that $C_{k} \cap C_{m}=\partial C_{k} \cap \partial C_{m}=v$ is a point.

b) The intersection of any three distinct 1-cells is empty.

2) $W$ is called a locally centered lump collection (LCL collection) if $W$ is a locally centered collection and a locally lump collection at the same time (Figure 1).

Collections of 1-cells shown in Figures 1(a)-(c) are LCL collections. (d) is not an LL collection because it violates condition (b) in Definition 2.2, i.e., $C_{3} \cap C_{4} \cap C_{5} \neq \varnothing$. (e) is not an LL collection because it violates condition (a) in Definition 2.2, i.e., $C_{4} \cap C_{5} \neq \partial C_{4} \cap \partial C_{5}$. (f) is not an LL collection because it violates condition (a) in Definition 2.2, i.e., $C_{1} \cap C_{2}=\partial C_{1} \cap \partial C_{2}=\left\{v_{1}, v_{2}\right\}$ is not a point. (g) is an LL but not LC collection.

From Definition 2.2, it follows that if $W=\left\{C_{1}, C_{2}, \cdots\right\}$ is an LCL collection of 1-cells, and the union $M=C_{1} \cup C_{2} \cup \cdots$ is a connected space, then $\mathrm{M}$ is either a circle or a segment or a line or a half line.

\section{Definition 2.3}

1) Let $W=\left\{D_{1}, D_{2}, \cdots\right\}$ be a collection of 2-cells. $W$ is called a locally lump collection (LL collection) if:

a) From condition $D_{k} \cap D_{m} \neq \varnothing, m \neq k$, it follows that $D_{k} \cap D_{m}=\partial D_{k} \cap \partial D_{m}=C_{k m}$ is a 1-cell.

b) From condition $D_{k} \cap D_{m} \cap D_{p} \neq \varnothing, m \neq k, m \neq p, k \neq p$, it follows that

$D_{k} \cap D_{m} \cap D_{p}=\partial D_{k} \cap \partial D_{m} \cap \partial D_{p}=C_{k m} \cap C_{k p}=\partial C_{k m} \cap \partial C_{k p}=v$ is a point.

c) The intersection of any four distinct 2-cells is empty.

2) $W$ is called a locally centered lump collection (LCL collection) if $W$ is a locally centered collection and a locally lump collection at the same time (Figure 2).

Collections of 2-cells are depicted in Figure 2. Collection (a) is an LC but not LL collection because nonempty intersections $D_{1} \cap D_{3}$ and $D_{2} \cap D_{4}$ are not 1-cells. Collection (b) is an LL but not LC collection because $D_{1} \cap D_{2} \neq \varnothing, D_{1} \cap D_{3} \neq \varnothing, D_{2} \cap D_{3} \neq \varnothing$ but $D_{1} \cap D_{2} \cap D_{3}=\varnothing$. Collection (c) is an LCL collection of 


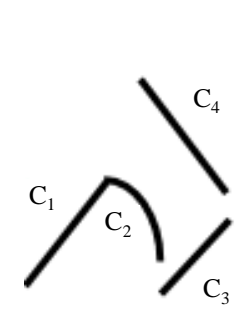

(a)

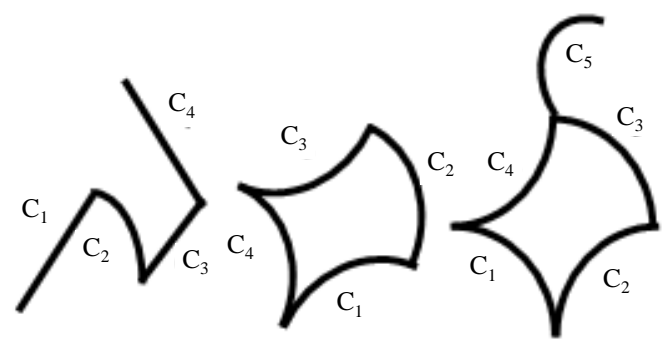

(c)

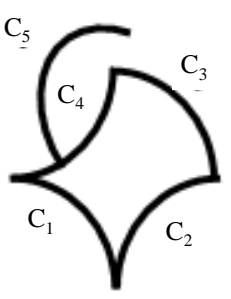

(e)

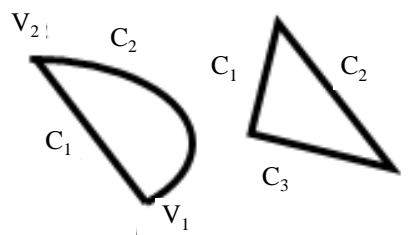

(f)

(g)

Figure 1. Collections of 1-cells $\mathrm{C}_{1}, \mathrm{C}_{2}, \ldots$ (a)-(c) are LCL collections. (d)-(f) are LC but not LL collections. (g) is an LL but not LC collection.

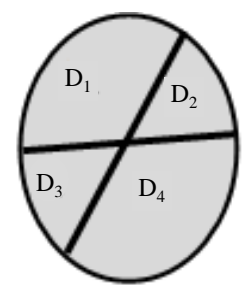

(a)

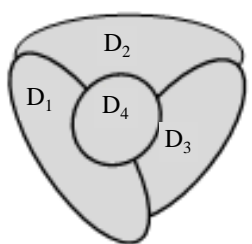

(b)

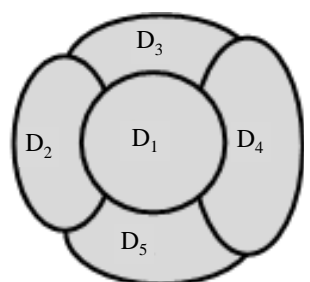

(c)

Figure 2. (a) is an LC but not an LL collection of 2-cells. (b) is an LL but not LC collection of 2-cells. (c) is an LCL collection of 2-cells.

2-cells. Collections $W_{1}, W_{2}$ and $W_{3}$ depicted in Figure 8 are LCL collections of 2-cells. The following corollary is a direct consequence of Definitions 2.2 and 2.3.

\section{Corollary 2.1}

Any subcollection of an LCL collection of 1-cells or 2-cells is an LCL collection.

In papers [8], locally centered collections were studied it was shown that for a given object, the intersection graphs of all continuous, regular and contractible covers are homotopic to each other. There is a significant number of papers which investigate collections of sets in 2D and 3D (see, e.g., [3]-[5] [9]-[12]). In papers [10]-[12], properties of SN sets were studied. A normal collection $W$ of convex non-generate polygons (intersection of any two of them is an edge, a vertex, or empty) was called strongly normal (SN) if for all $P$, $P_{1}, \cdots, P_{n}(n>0) \in W$, if each $P_{i}$ intersects $P$ and $I=P_{1} \cap \cdots \cap P_{n}$ is non-empty, then $I$ intersects $P$ ). In paper [4], it was shown that cells in strongly normal collections can be arbitrary topological disks.

The advantage of using LCL covers in discretization schemes is that the digital models of continuous objects have the same topology as their continuous counterparts. For example, if we use an LCL cover of the plane, then the digital model of the plane is necessarily a digital 2-surface, i.e., an LCL discretization scheme preserves the topology of the plane. Besides, cells in an LCL collection can be of an arbitrary shape and size. This property allows us to use a fine resolution (with any necessary small size of cells) in areas of interest, where image details are required, and a coarse resolution in the other parts of the object.

Regard now the set of 2-cells intersecting a given 2-cell in an LCL collection. This set specifies local topological properties of the collection.

\section{Proposition 2.1}

Let $W=\left\{D_{0}, D_{1}, \cdots\right\}$ be an LCL collection of 2-cells, $U=\left\{D_{1}, D_{2}, \cdots, D_{s}\right\}$ be a collection of all 2-cells intersecting $D_{0}$, and $V=\left\{C_{1}, C_{2}, \cdots, C_{s}\right\}$ be the collection of 1-cells $C_{i}=D_{0} \cap D_{i} \neq \varnothing, i=1, \cdots, s$. Then $V$ is an LCL collection.

\section{Proof}

Consider the collection $V=\left\{C_{1}, C_{2}, \cdots, C_{s}\right\}$ of 1-cells. Suppose that $C_{k} \cap C_{i} \neq \varnothing$. Then $C_{k} \cap C_{i}=\partial C_{k} \cap \partial C_{i}=\left(D_{0} \cap D_{i}\right) \cap\left(D_{0} \cap D_{k}\right)=D_{0} \cap D_{i} \cap D_{k} \neq \varnothing$. Since W is an LCL collection, then $D_{0} \cap D_{i} \cap D_{k}=C_{k} \cap C_{i}=\partial C_{k} \cap \partial C_{i}=v$ is a point according to Definition 2.3. Consider the intersection $C_{k} \cap C_{m} \cap C_{p}, m \neq k, m \neq p, k \neq p$. Then $C_{k} \cap C_{m} \cap C_{p}=D_{k} \cap D_{m} \cap D_{p} \cap D_{0}$. Since W is an LCL collection, then $D_{k} \cap D_{m} \cap D_{p} \cap D_{0}=\varnothing$ according to Definition 2.3. Therefore, $C_{k} \cap C_{m} \cap C_{p}=\varnothing$. Thus, $V=\left\{C_{1}, C_{2}, \cdots, C_{s}\right\}$ is an LCL collection of 1-cells according to Definition 2.2. $\square$ 
In order to prove the following proposition, let us remind the definition of isomorphic sets. A collection $W=\left\{A_{0}, A_{1}, \cdots\right\}$ of sets is isomorphic to a collection $V=\left\{B_{0}, B_{1}, \cdots\right\}$ of sets, if there exists one-one onto correspondence $f: W \rightarrow V$ such that

$A_{i} \cap \cdots \cap A_{k} \cap \cdots \cap A_{p} \neq \varnothing$ if and only if $f\left(A_{i}\right) \cap \cdots \cap f\left(A_{k}\right) \cap \cdots \cap f\left(A_{p}\right) \neq \varnothing$.

\section{Proposition 2.2}

Let $W=\left\{D_{0}, D_{1}, \cdots\right\}$ be an LCL collection of 2-cells, $U=\left\{D_{1}, D_{2}, \cdots, D_{s}\right\}$ be a collection of all 2-cells intersecting $D_{0}$ and $V=\left\{C_{1}, C_{2}, \cdots, C_{s}\right\}$ be an LCL collection of 1-cells $C_{i}=D_{0} \cap D_{i} \neq \varnothing, i=1, \cdots, s$. Then collections $U=\left\{D_{1}, D_{2}, \cdots, D_{s}\right\}$ and $V=\left\{C_{1}, C_{2}, \cdots, C_{s}\right\}$ are isomorphic.

Proof

Let $U=\left\{D_{1}, D_{2}, \cdots, D_{s}\right\}, V=\left\{C_{1}, C_{2}, \cdots, C_{s}\right\} . D_{i} \in U, D_{k} \in U, i \neq k$, and $D_{i} \cap D_{k} \neq \varnothing$.

Suppose that $D_{i} \in U, D_{k} \in U, i \neq k$, and $D_{i} \cap D_{k} \neq \varnothing$. Since $D_{0} \cap D_{i} \neq \varnothing, D_{0} \cap D_{k} \neq \varnothing$, and $W$ is an LCL collection, then $D_{0} \cap D_{i} \cap D_{k} \neq \varnothing$. Since $C_{i} \cap C_{k}=D_{0} \cap D_{i} \cap D_{k}$, then $C_{i} \cap C_{k} \neq \varnothing$. Suppose that $D_{i} \in U, D_{k} \in U, D_{p} \in U, i \neq k, i \neq p, p \neq k$, and consider the intersection $D_{p} \cap D_{i} \cap D_{k}$. Since $W$ is an LCL collection, then $D_{p} \cap D_{i} \cap D_{k}=D_{p} \cap D_{i} \cap D_{k} \cap D_{0}=\varnothing$.

Since $C_{p} \cap C_{i} \cap C_{k}=D_{p} \cap D_{i} \cap D_{k} \cap D_{0}$, then $D_{p} \cap D_{i} \cap D_{k}=\varnothing$. Similarly, if $C_{i} \cap C_{k} \neq \varnothing$, then $D_{i} \cap D_{k} \neq \varnothing$, and since $C_{p} \cap C_{i} \cap C_{k}=\varnothing$, then $D_{p} \cap D_{i} \cap D_{k}=\varnothing$. Therefore, $f: U \rightarrow V$ such that $f\left(D_{i}\right)=C_{i}$, $i=1, \cdots, s$, is an isomorphism.

The number of elements in an LCL collection can be reduced by merging couples of adjacent 1-cells or 2cells.

\section{Proposition 2.3}

Let $W=\left\{C_{0}, C_{1}, \cdots\right\}$ be an LCL collection of 1-cells, $C_{0} \cap C_{1} \neq \varnothing$ and $B=C_{0} \cup C_{1}$. Let $V=\left\{B, C_{2}, \cdots\right\}$ be a collection of 1-cells obtained by merging $C_{0}$ and $C_{1}, C_{i}$ is adjacent to $C_{0}, C_{k}$ is adjacent to $C_{1}$.

- If $C_{i} \cap C_{k}=\varnothing$, then $V=\left\{B, C_{2}, \cdots\right\}$ is an LCL collection of 1-cells (Figure 3(a), Figure 3(b)).

- If $C_{i} \cap C_{k} \neq \varnothing$, then $V=\left\{B, C_{2}, \cdots\right\}$ is not an LCL collection of 1-cells (Figure 3(c), Figure 3(d)).

\section{Proof}

Suppose $C_{i} \cap C_{k}=\varnothing$. Then $B \cap C_{i}=C_{0} \cap C_{i}=v_{1}$, and $B \cap C_{k}=C_{0} \cap C_{k}=v_{2}$, are points, and $V$ is an LCL collection of 1-cells by construction of $V$ (see Figure 3(a), Figure 3(b)).

Suppose $C_{i} \cap C_{k}=\varnothing$. By construction of $V=\left\{C_{i}, B, C_{k}\right\}, C_{i} \cap C_{k} \neq \varnothing, C_{i} \cap B \neq \varnothing, B \cap C_{k} \neq \varnothing$. Since $B \cap C_{i} \cap C_{k}=\varnothing$, then $V$ is not a locally centered collection (see Figure 3(c), Figure 3(d)). Thus, $\mathrm{V}$ is not an LCL collection.

\section{Proposition 2.4}

Let $W=\left\{D_{0}, D_{1}, \cdots\right\}$ be an LCL collection of 2-cells, $D_{0} \cap D_{1} \neq \varnothing$ and $B=D_{0} \cup D_{1}$. Let $V=\left\{B, D_{2}, \cdots\right\}$ be a collection of 2-cells obtained by merging $D_{0}$ and $D_{1}$.

- If for any $D_{i}$ such that $D_{0} \cap D_{i} \neq \varnothing, D_{1} \cap D_{i}=\varnothing$, and for any $D_{k}$ such that $D_{1} \cap D_{k} \neq \varnothing, D_{0} \cap D_{k}=\varnothing$, it follows that $D_{k} \cap D_{i}=\varnothing$, then $V=\left\{B, D_{2}, \cdots\right\}$ is an LCL collection of 2-cells (Figure 4(a), Figure 3(b)).

- If there are 2-cells $D_{i}$ and $D_{k}$ such that $D_{0} \cap D_{i} \neq \varnothing, D_{1} \cap D_{i}=\varnothing, D_{1} \cap D_{k} \neq \varnothing, D_{0} \cap D_{k}=\varnothing$, and $D_{k} \cap D_{i} \neq \varnothing$, then $V=\left\{B, D_{2}, \cdots\right\}$ is not an LCL collection of 2-cells (Figure 4(c)).

\section{Proof}

Suppose that $B \cap D_{i} \neq \varnothing, B \cap D_{k} \neq \varnothing$ and $D_{k} \cap D_{i} \neq \varnothing$. Since $W$ is an LCL collection, there can be only constructions depicted in Figures 4(a)-(c). For simplicity, we omit cases obtained by replacing indexes " 0 " with "1", " 1 " with "0", " $I$ " with " $k$ " and " $k$ " with "I".

Consider an LCL collection $W=\left\{D_{0}, D_{1}, \cdots\right\}$ and the collection $V=\left\{B, D_{2}, \cdots\right\}$ depicted in Figure 4(a).

Since $B \cap D_{i} \cap D_{k}=v, B \cap D_{k}=C_{k 0}$, then $V$ is an LCL collection. Consider an LCL collection $W=\left\{D_{0}, D_{1}, \cdots\right\}$ and the collection $V=\left\{B, D_{2}, \cdots\right\}$ depicted in Figure 4(b). It follows from construction of $V$ that $B \cap D_{i} \cap D_{k}=v . B \cap D_{k}=C_{k 0} \cup C_{k 1}$, i.e., $V$ is an LCL collection. Consider an LCL collection $W=\left\{D_{0}, D_{1}, \cdots\right\}$ and the collection $V=\left\{B, D_{2}, \cdots\right\}$ depicted in Figure 4(c). By construction of $V$, $B \cap D_{i} \neq \varnothing, \quad B \cap D_{k} \neq \varnothing, \quad D_{k} \cap D_{i} \neq \varnothing$, but $B \cap D_{i} \cap D_{k}=\varnothing$. Therefore, $V$ is not an LCL collection. $\square$

\section{Digital 1- and 2-Surfaces and Digital Models of the Plane}

Traditionally, a digital image has the structure of a simple graph $G=(V, E)$, where $V=\left\{v_{1}, v_{2}, \cdots, v_{n}, \cdots\right\}$ is a set of vertices, and $E=\left\{\left(v_{p} v_{q}\right), \cdots\right\} \subseteq V \times V$ is a set of edges. Edges between vertices define the nearness in the neighborhood of any vertex and such notions as the connectedness, the dimensionality and the distance on $G$ are 


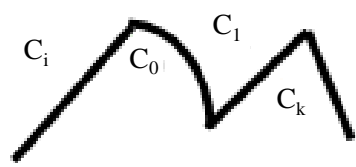

(a)

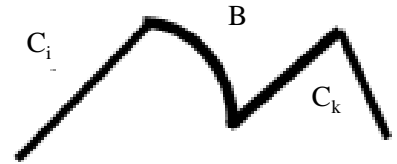

(b)

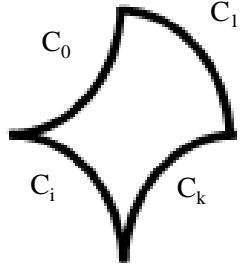

(c)

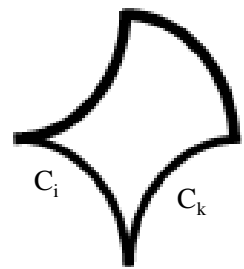

(d)

Figure 3. (a) An LCL collection W of 1-cells. (b) An LCL collection V of 1-cells. (c) An LCL collection W of 1-cells. (d) Collection V is not an LCL collection.

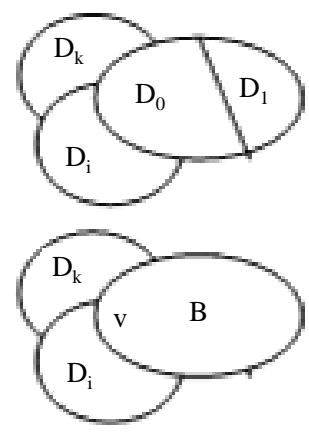

(a)

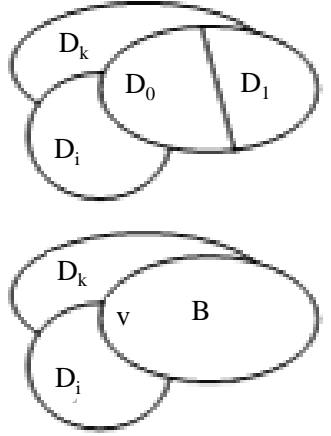

(b)

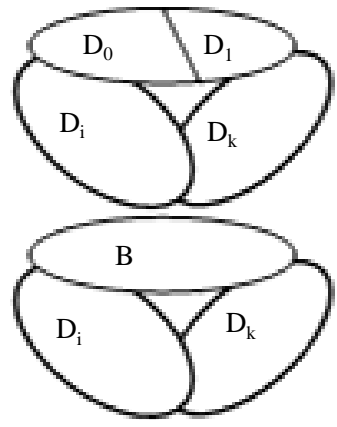

(c)

Figure 4. (a) W is an LCL collection. V is an LCL collection. (b) W is an LCL collection. V is an LCL collection. (c) $\mathrm{W}$ is an LCL collection. V is not an LCL collection.

completely defined by sets $V$ and $E$ (see, e.g., [6]-[9] [13] [14]).

We use the notations $v_{p} \in G$ and $\left(v_{p} v_{q}\right) \in G$ if $v_{p} \in V$ and $\left(v_{p} v_{q}\right) \in E$ respectively if no confusion can result.

For two graphs $G=(V, E)$ and $H=(Y, W)$ with disjoint vertex sets $V$ and $Y$, their join $G \oplus H$ is the graph that contains $G, H$, and edges joining every vertex in $G$ with every vertex in $H$.

An induced subgraph $O(v) \subseteq G$ containing all vertices of $G$ adjacent to $v$ is called the rim or the neighborhood of $v$ in $G$, the subgraph $U(v)=\{v\} \oplus O(v)$ is called the ball of $v$ [14]. If $G=\{v\}$ is a singleton, we write $v$ rather than $\{v\}$ if no confusion can result.

Two graphs $G$ and $H$ are called homotopy equivalent if one of them can be converted to the other one by a sequence of contractible transformations. Properties of contractible transformations were studied in [9] [14]. In particular, it was shown that contractible transformations do not change the Euler characteristic and the homology groups of graphs.

If we consider a graph as a digital space, we use the word space for a graph and the word point for a vertex. Notice that in this paper, a subspace is an induced subspace. In papers [9] [13], digital n-surfaces and n-manifolds were defined and investigated.

\section{Definition 3.1}

- A digital 0-dimensional surface (sphere) is a graph $S^{0}(x, y)$ with just two non-adjacent points $x$ and $y$.

- A connected space $M$ is called a digital 1-surface, if for any point $v \in M$, the rim $O(v)$ is a digital 0-dimensional surface.

A digital 1-surface has four or more points. Digital 1-spheres $G_{0}-G_{3}$ are depicted if Figure 5. A digital line $G_{4}$ has an infinite number of points (see Figure 5).

Consider a sequence of transformations, which reduce the number of points in a given digital 1-surface $G$. In graph theory, the contraction of points $x$ and $y$ in a graph $G$ is the replacement of $x$ and $y$ with a point $\mathrm{z}$ such that zis adjacent to the points to which points $x$ and $y$ were adjacent. The resulting graph has one point less than $G$. Therefore, $G$ must contain two adjacent points $\mathrm{x}$ and $y$ such that after their contraction, the obtained space 
$N=(G-\{x, y\}) \cup z$ is a digital 1-surface, which is homotopy equivalent to $G$. For example, a minimal digital 1-sphere is the join $S_{\min }^{1}=S^{0}\left(u_{1}, v_{1}\right) \oplus S^{0}\left(u_{2}, v_{2}\right)$ of two copies of a zero-dimensional sphere $\left(G_{5}\right.$ in Figure 5$)$ [9] [13]. A digital 1-surface $M$ is called a digital 1-sphere (circle) if it can be converted to $S_{\min }^{1}$ by a sequence of contracting adjacent points.

Definition 3.2

Let $\mathrm{M}$ be a digital 1-surface and $\mathrm{x}$ and $\mathrm{y}$ be adjacent points of $M$. We say that $\{x, y\}$ is a simple pair if the space $U(x) \cup U(y)-\{x, y\}$ is a digital 0 -sphere.

In Figure 6(a), $\{x, y\}$ is a simple pair.

Proposition 3.1

Let $\{x, y\}$ be a simple pair lying in a digital 1-surface $M$. Then the space $N=(M-\{x, y\}) \cup z$, obtained by contraction of $\{x, y\}$ is a digital 1-surface.

Proof

The proof is depicted in Figure 6. Since $\{x, y\}$ is a simple pair of points, then $U(x) \cup U(y)-\{x, y\}=\{v, u\}$ a digital 0-sphere (Figure 6(a)). Glue a point $\mathrm{z}$ to $\mathrm{M}$ in such a way that $O(z)=U(x) \cup U(y)$, and delete points $x$ and $y$ (Figure 6(b), Figure 6(c)). By construction, the obtained space $N=(M-\{x, y\}) \cup z$ is a digital 1surface. The proof is complete. $\square$

Remark 3.1

It is easy to see from Proposition 3.3, that a digital 1-surface with a finite number of points is a digital 1-sphere, which can be transformed to a minimal digital 1-sphere by sequential contraction of simple pairs. Digital 1-surfaces $G_{1}-G_{3}$ depicted in Figure 5 can be transformed to a minimal 1-sphere $G_{0}$ by sequential contracting simple pairs. Thus, a digital 1-surface is either a digital 1-sphere or a digital line.
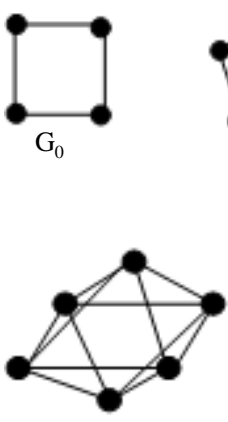

$\mathrm{G}_{5}$
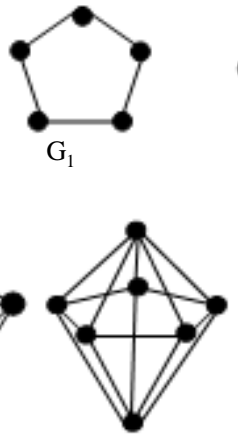

$\mathrm{G}_{6}$
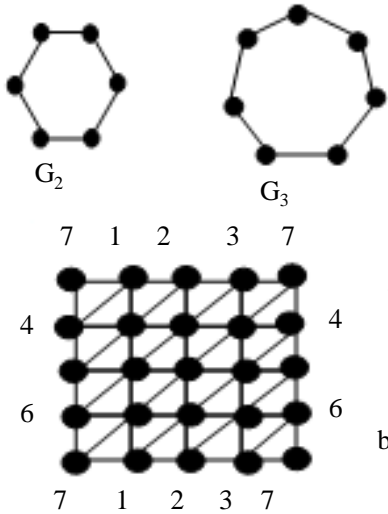

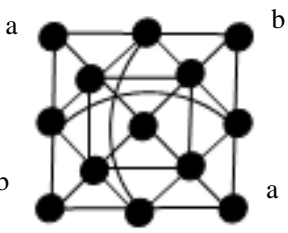

$\mathrm{G}_{8}$

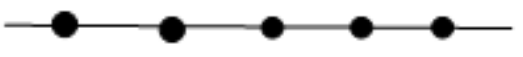

$\mathrm{G}_{4}$

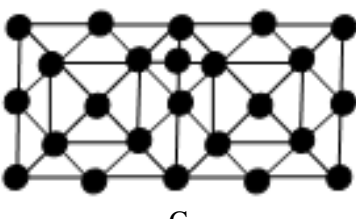

$\mathrm{G}_{9}$

Figure 5. $G_{0}-G_{3}$ are digital closed 1-surfaces (digital circles). $G_{4}$ is the digital line. $G_{5}-G_{8}$ are digital closed 2-surfaces. $\mathrm{G}_{9}$ is a portion of the digital plane.

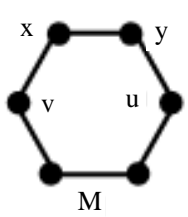

(a)

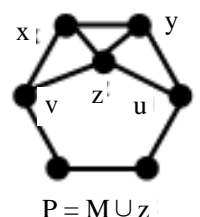

(b)

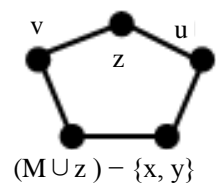

(c)

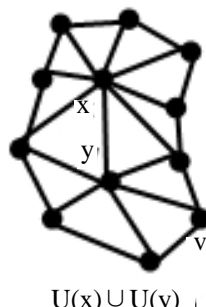

(d)

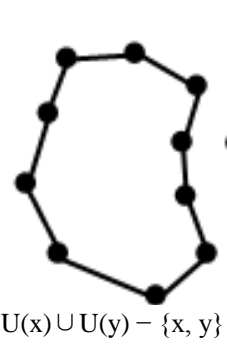

(e)

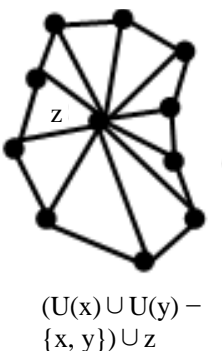

(f)

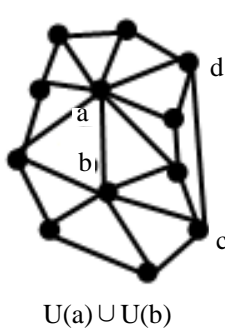

(g)

Figure 6. (a) $\mathrm{M}$ is a digital 1-sphere with six points. (b) $\{\mathrm{x}, \mathrm{y}\}$ is a topological pair of points. (c) $N=(M \cup z)-\{x, y\}$ is a digital 1-sphere with five points. (d)-(f) $U(x) \cup U(y)$ is a contractible space. $U(x) \cup U(y)-\{x, y\}$ is a digital 1 -sphere. $\{\mathrm{x}, \mathrm{y}\}$ is a topological pair of points. (g) $\{\mathrm{a}, \mathrm{b}\}$ is not a topological pair of points. $U(a) \cup U(b)-\{a, b\}$ is not a digital 1-sphere. 


\section{Definition 3.3}

- A connected digital space $M$ is called a digital 2-surface if for any point $v$ of $M$, the rim $O(v)$ is a digital 1sphere [13].

A digital 2-surface with a finite number of points is called a digital closed 2-surface. In Figure 5, $G_{5}-G_{8}$ are digital closed 2-surfaces. $G_{9}$ is a portion of a digital plane.

Consider a sequence of transformations, which reduce the number of points in a given digital 2-surface $M$. As above, this is a contraction of two adjacent points $x$ and $y$ such that after their contraction, the obtained space $N=(M-\{x, y\}) \cup z$ is a digital 2-surface.

Definition 3.4

Let $M$ be a digital 2-surface and $x$ and $y$ be adjacent points of $M$. We say that $\{x, y\}$ is a simple pair if the space

$U(x) \cup U(y)-\{x, y\}$ is a digital 1-sphere (see Figure 6(d), Figure 6(e)).

In Figure 6(d), $\{x, y\}$ is a simple pair. A pair $\{a, b\}$ depicted in Figure 6(g) is not a simple pair.

Proposition 3.2

Let $\{x, y\}$ be a simple pair lying in a digital 2-surface $M$. Then the space $N=(M-\{x, y\}) \cup z$ obtained by contraction of $\{x, y\}$ is a digital 2-surface.

\section{Proof}

The proof is similar to the proof of Proposition 3.3. Delete from $M$ points $x$ and $y$ and glue a point $z$ in such a way that $O(z)=U(x) \cup U(y)-\{x, y\}$. Therefore, the rim of point $z$ is a digital 1-surface according to Definition 3.6 (see Figure 6(f)). The rims of all other points belonging to $N$ are digital 1-surfaces by construction. Therefore, $N$ is a digital 2-surface. The proof is complete. $\square$

The join $S_{\text {min }}^{2}=S^{0}\left(u_{1}, v_{1}\right) \oplus S^{0}\left(u_{2}, v_{2}\right) \oplus S^{0}\left(u_{3}, v_{3}\right)$ of three copies of a zero-dimensional sphere is a minimal digital 2-sphere ( $G_{5}$ in Figure 5). A digital 2-surface $M$ is called a digital 2-sphere if it is homotopy equivalent to a minimal digital 2-sphere [8] [9]. It can be checked directly that a digital 2-sphere $\mathrm{G}_{6}$ depicted in Figure 5 can be converted to $G_{5}$ by a sequential contraction of simple pairs. $G_{7}$ is a digital torus, and $G_{8}$ is a digital projective plane. $G_{7}$ and $G_{8}$ have no simple pairs, and cannot be converted to $G_{5}$ by sequential contractions of simple pairs. Properties of a digital sphere, a digital torus and a digital projective plane were studied in [8] [9].

Digital 2-surfaces can be used to construct topologically correct digital models of segmented medical images.

\section{Digital Models of LCL Collections and Digital Models of the Plane}

Let us investigate a connection between a segmented image of an object of interest and the digital model of this image. We are going to show that an LCL segmentation of an object incorporates topological features directly into the segmentation process.

Remind the definition of the intersection graph of a family of sets. Let $W=\left\{D_{1}, D_{2}, \cdots, D_{n}, \cdots\right\}$ be a finite or countable family of sets. Then the graph $G(W)$ with points $\left\{x_{1}, x_{2}, \cdots, x_{n}, \cdots\right\}$ is called the intersection graph of $W$, if points $x_{k}$ and $x_{i}$ are adjacent whenever $D_{k} \cap D_{i} \neq \varnothing$. In other word, $f: G(W) \rightarrow W$ such that $f\left(x_{i}\right)=D_{i}$ is an isomorphism.

\section{Definition 4.1}

- Let $W=\left\{D_{1}, D_{2}, \cdots\right\}$ be an LCL collection of 1-cells or 2-cells, and $M$ be the union of all cells, $M=D_{1} \cup D_{2} \cup \cdots$. Then the collection $W$ is called an $L C L$ cover of $M$.

- Let $W=\left\{D_{1}, D_{2}, \cdots\right\}$ be an LCL collection of 1-cells or 2-cells, and $G(W)$ be the intersection graph of $W$. Then $G(W)$ is called the digital model of $M=D_{1} \cup D_{2} \cup \cdots$ in regard to $W$ (Figure 7, Figure 8).

\section{Theorem 4.1}

Let $M$ be a circle and $W=\left\{C_{0}, C_{1}, \cdots, C_{n}\right\}$ be an LCL cover of $M=C_{0} \cup \cdots \cup C_{n}$ by 1-cells. Then the digital model $G(W)$ of $M$ in regard to $W$ is a digital 1-surface.

\section{Proof}

Let $W=\left\{C_{0}, C_{1}, \cdots, C_{n}\right\}$ be an LCL cover of a circle $M$ by 1-cells such that $C_{k} \cap C_{i} \neq \varnothing$ if $k=(i+1) \bmod (n+1)$. Let $G(W)=\left\{x_{0}, x_{1}, \cdots, x_{n}\right\}$ be the intersection graph of $W$, where $x_{i}=f\left(C_{i}\right)$ (Figures 7(a)-(c)). This means that points $x_{k}$ and $x_{i}$ are adjacent whenever $C_{k} \cap C_{i} \neq \varnothing$. Consider 1-cell $C_{1}$. Then $C_{1} \cap C_{0}=v_{0}$ and $C_{1} \cap C_{2}=v_{2}$ are endpoints of $C_{1}, C_{1} \cap C_{i}=\varnothing, i \neq 0,2$, and $C_{0} \cap C_{2}=\varnothing$. Therefore, point $x_{1}$ is adjacent only to points $x_{0}$ and $x_{2}$ and points $x_{0}$ and $x_{2}$ are non-adjacent. Therefore, the rim $O\left(x_{1}\right)$ in $G(W)$ is a digital 0 -surface consisting of two non-adjacent points $x_{0}$ and $x_{2}$. For the same reason, the rim of any point in 


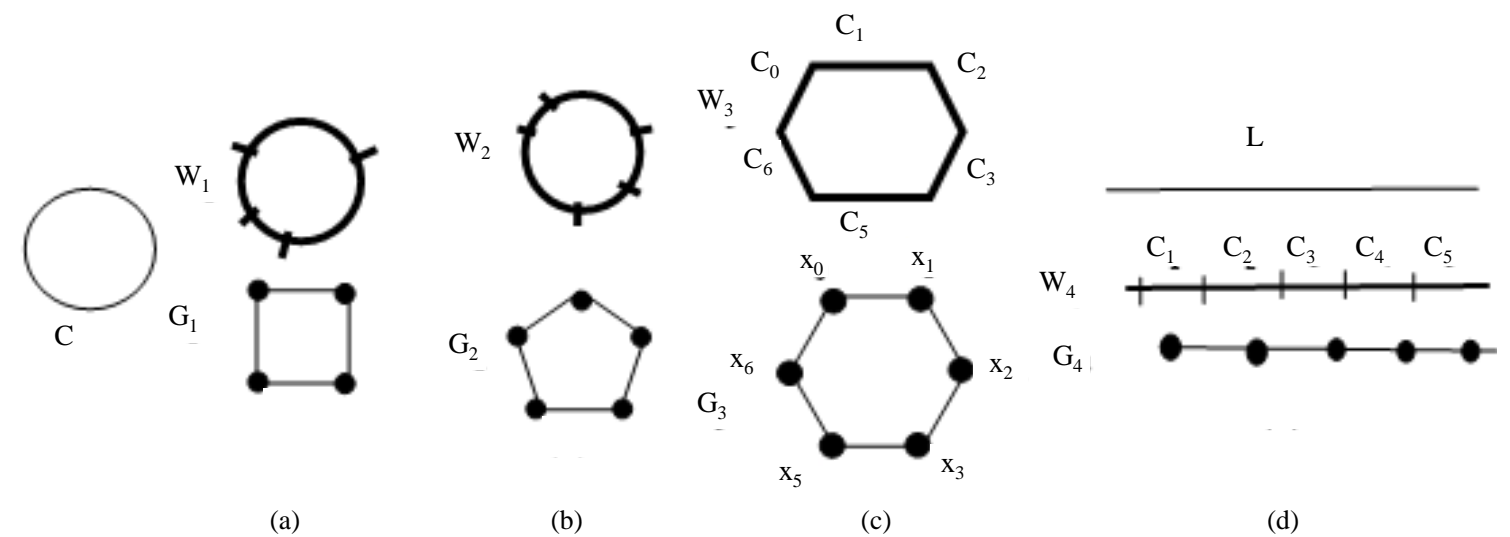

Figure 7. (a), (b) and (c) $\mathrm{W}_{1}, \mathrm{~W}_{2}, \mathrm{~W}_{3}$ are LCL covers of a circle $C, \mathrm{G}_{1}, \mathrm{G}_{2}, \mathrm{G}_{3}$ are the corresponding digital models of C. (d) $\mathrm{W}_{4}$ is an LCL cover of line $\mathrm{L}, \mathrm{G}_{4}$ is the corresponding digital model of $\mathrm{L}$.

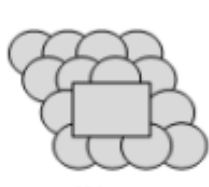

$\mathrm{W}_{1}$

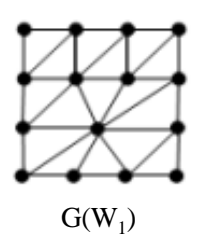

$\mathrm{G}\left(\mathrm{W}_{1}\right)$

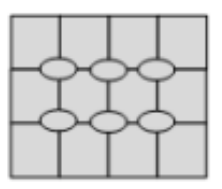

$\mathrm{W}_{2}$

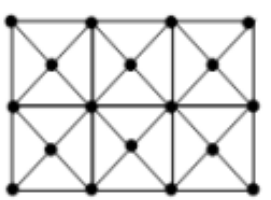

$\mathrm{G}\left(\mathrm{W}_{2}\right)$

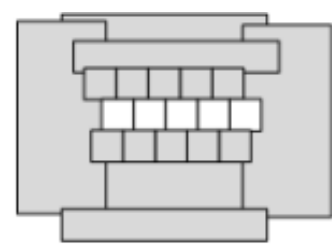

$\mathrm{W}_{3}$

Figure $8 . W_{1}, W_{2}$ and $W_{3}$ are LCL covers of the plane $E^{2} . G\left(W_{1}\right), G\left(W_{2}\right)$ are digital models of $E^{2}$.

$G(W)$ is a digital 0-surface. Hence, $G(W)$ is a digital 1-surface according to Definition 3.1.

In Figure 7, $W_{1}, W_{2}$ and $W_{3}$ are LCL covers of a circle $C$, and $G_{1}, G_{2}$ and $G_{3}$ are their intersection graphs. Evidently, $G_{1}, G_{2}$ and $G_{3}$ are digital 1-surfaces according to Definition 3.1. $W_{4}$ is an LCL cover of a line L. The intersection graph $G_{4}$ of $W_{4}$ is a digital 1-surface. A cover $V=\left\{C_{1}, C_{2}\right\}$ of a circle shown in Figure 3(c) is not an LCL cover, and the intersection graph $G(V)$ containing two adjacent points is not a digital 1-surface. Similarly, a cover $U=\left\{C_{1}, C_{2}, C_{3}\right\}$ of a circle shown in Figure 3(d) is not an LCL cover, and the intersection graph $G(U)$ containing three adjacent points is not a digital 1-surface.

\section{Theorem 4.2}

Let $M$ be a circle, $W=\left\{C_{0}, C_{1}, C_{2}, \cdots, C_{n}\right\}$ be an LCL cover of $M=C_{0} \cup \cdots \cup C_{n}$ by 1-cells. and $G(W)=\left\{x_{0}, x_{1}, x_{2}, \cdots, x_{n}\right\}$ be a digital model of $M$ in regard to $W$. Let $V=\left\{C_{1}, B, C_{3}, \cdots, C_{n}\right\}$ be the cover of $M$ by 1-cells obtained by merging $C_{1}$ and $C_{2}, B=C_{1} \cup C_{2}$, and $G(V)=\left\{x_{0}, z, x_{3}, \cdots, x_{n}\right\}$ be the digital model of $M$ in regard to $V$.

If $V=\left\{C_{1}, B, C_{3}, \cdots, C_{n}\right\}$ is an LCL cover of $M$, then $\left\{x_{1}, x_{2}\right\}$ is a simple pair in the digital 1-surface $G(W)$ and $G(V)=\left(G(W)-\left\{x_{1}, x_{2}\right\}\right) \cup z$ is a digital 1-surface obtained from $G(W)$ by contracting $\left\{x_{1}, x_{2}\right\}$.

\section{Proof}

According to Proposition 2.3, $C_{0} \cap C_{3}=\varnothing$. Therefore, points $x_{0}$ and $x_{3}$ are not adjacent and $\left\{x_{1}, x_{2}\right\}$ is a simple pair in $G(W)$. This means that the rim $O(z)$ is a digital 0-sphere $S_{\min }^{0}\left(x_{1}, x_{3}\right)$. The rims of all other points of $G(V)$ are also digital 0 -spheres by construction of $G(V)$. $\square$

A collection $W_{2}$ depicted in Figure 7(b) is an LCL cover of a circle $C$. Any pair of adjacent 1-cells of $W_{2}$ is a simple pair. By merging any two adjacent 1-cells, $W_{2}$ can be transformed to an LCL cover $W_{1}$ containing four elements (Figure 7(a)). Similarly, the intersection graph $G_{2}$ containing a simple pair of points can be transformed to a 1-surface $G_{1}$ by contracting these points (see Figure 7(a) and Figure 7(b)). An LCL cover $W_{2}$ is a minimal LCL cover of a circle, and $G_{2}$ does not contain simple pairs (see Figure 7(a)).

In paper [8], the intersection graphs of covers of convex 2-cells were studied. Let $F=\left\{U_{1}, U_{2}, \cdots, U_{n}, \cdots\right\}$ be a set of all unit squares on $E^{2}$ whose vertices are located in points with integer coordinates, and whose edges are parallel to the coordinate exes. $F$ is a cover of $E^{2}$. Suppose that $M$ is a convex 2-cell on $E^{2}$, and

$W=\left\{U_{1}, U_{2}, \cdots, U_{n}\right\}$ is a collection of all squares intersecting $M$. It was shown that the intersection graph $G(W)$ of $W$ is contractible, i.e., can be converted to a one-point graph by a sequence of contractible transformations. 
Using this result and the notion of isomorphic covers it was shown that the intersection graphs of covers of a sphere, a torus and a projective plane are homotopy equivalent to digital 2-surfaces $G_{5}, G_{7}$ and $G_{8}$ respectively depicted in Figure 5.

\section{Theorem 4.3}

Let $M=E^{2}$ be the plane, and $W=\left\{D_{0}, D_{1}, \cdots\right\}$ be an LCL cover of $M=D_{0} \cup D_{1} \cup \cdots$ by 2-cells such that for any $D_{i} \in W$, the collection of all 2-cells of $W$ intersecting $D_{i}$ is finite. Then the digital model $G(W)$ of $M$ is a digital 2-surface.

Proof

Suppose that $W=\left\{D_{0}, D_{1}, \cdots\right\}$ is an LCL cover of $M$, and $G(W)$ is the intersection graph of $W$ such that $x_{i}=f\left(D_{i}\right), i=0,1, \cdots$. Consider the collection $U=\left\{D_{1}, D_{2}, \cdots, D_{s}\right\}$ containing all 2-cells which intersect $D_{0}$. Then the collection $V=\left\{C_{1}, C_{2}, \cdots, C_{s}\right\}$, where $C_{i}=D_{0} \cap D_{i}, i=1, \cdots, s$, is an LCL collection of 1-cells, and collections $U$ and $V$ are isomorphic to each other according to Proposition 2.2. Therefore, the intersection graph $G(U), G(U) \subseteq G(W)$, of collection $U$ is the intersection graph $G(V)$ of collection $V$, where $x_{i}=f\left(C_{i}\right), i=1, \cdots, s$. Since $G(V)$ is a digital 1-sphere according to Theorem 3.1, then $G(U)$ is a digital 1-sphere. Therefore, the rim $O\left(x_{0}\right)=G(U)$ of $x_{0}$ in $G(W)$ is a digital 1-sphere. For the same reason, the rim of any point in $G(W)$ is a digital 1-sphere. Hence, $G(W)$ is a digital 2-surface according to Definition 3.3.

Figure 8 contains LCL covers $W_{1}, W_{2}$ and $W_{3}$ of the plane $E^{2}$ and digital models $G\left(W_{1}\right)$ and $G\left(W_{2}\right)$ which are digital 2-surfaces. One can check directly that $G\left(W_{3}\right)$ is also a digital 2-surface.

Consider now the replacement of two adjacent 2-cells with their union. Find conditions which guarantee that a new cover of $E^{2}$ is an LCL cover.

\section{Theorem 4.4}

Let $M=E^{2}$ be the plane, $W=\left\{D_{0}, D_{1}, \cdots\right\}$ be an LCL cover of $M=D_{0} \cup D_{1} \cup \cdots$ by 2-cells and $G(W)=\left\{x_{0}, x_{1}, \cdots\right\}$ be a digital model of $\mathrm{M}$ in regard to $W$. Let $V=\left\{D_{0}, B, D_{3}, \cdots\right\}$ be the cover of $M=D_{0} \cup B \cup D_{3} \cup \cdots$ by 2-cells obtained by merging $D_{1}$ and $D_{2}, B=D_{1} \cup D_{2}$, and $G(V)=\left\{x_{0}, z, x_{3}, \cdots\right\}$ be the digital model of $M$ in regard to $V$. If for any $D_{i}$ such that $D_{0} \cap D_{i} \neq \varnothing, D_{1} \cap D_{i}=\varnothing$, and for any $D_{k}$ such that $D_{1} \cap D_{k} \neq \varnothing, D_{0} \cap D_{k}=\varnothing$, it follows that $D_{i} \cap D_{k}=\varnothing$, then $\left\{x_{1}, x_{2}\right\}$ is a simple pair in the digital 2-surface $G(W)$ and $G(V)=\left(G(W)-\left\{x_{1}, x_{2}\right\}\right) \cup z$ is a digital 2-surface obtained from $G(W)$ by contracting $\left\{x_{1}\right.$, $\left.x_{2}\right\}$.

\section{Proof}

According to Proposition 2.4, $V=\left\{D_{1}, B, D_{3}, \cdots\right\}$ is an LCL collection of 2-cells. Therefore, the rim $O(z)$ of point $z$ in $G(V)$ is a digital 1-sphere. Since $U\left(x_{2}\right) \cup U\left(x_{1}\right)-\left\{x_{1}, x_{2}\right\}=O(z)$ is a digital 1-sphere, then $\left\{x_{0}, x_{1}\right\}$ is a simple pair according to Definition 3.4 and $G(V)=\left(G(W)-\left\{x_{1}, x_{2}\right\}\right) \cup z$, is a digital 2-surface obtained from $G(W)$ by contracting $\left\{x_{1}, x_{2}\right\}$. The proof is complete.

It is easy to check directly (see Figure 8) that merging any pair of adjacent 2-cells converts $W_{2}$ to non-LCL covers of the plane. Similarly, the intersection graph $G\left(W_{2}\right)$ do not contain simple pairs. In $W_{3}$, all white 2-cells can be merged together and the obtained cover is an LCL collection.

In paper [7], Eckhardt and Latecki studied digital topologies in $Z^{2}\left(Z^{2}\right.$ is the set of points with integer coordinates in $E^{2}$ ). They showed that "there are only two topologies in $Z^{2}$ whose connected sets are connected in the intuitive sense.” In the present paper, we do not use $Z^{2}$ as a basic set of points for building a topology. In this paper, a digital point corresponds to a 2-cell in an LCL cover of the continuous plane. Since elements of an LCL cover can be chosen arbitrarily (within LCL cover), then there are infinitely many non-isomorphic digital models of the plane.

As one can see from Figure 8, 2-cells have different shapes and sizes within the same LCL cover. There is a direct similarity between the plane and its digital models which are digital 2-surfaces. In $E^{2}$, an open neighborhood of a point x contains an open 2-disk $D$ whose boundary is a circle $C$. In $G\left(W_{1}\right)$ and $G\left(W_{2}\right)$, a point v belongs to the ball $U(v)=\{v\} \oplus O(v)$ whose boundary is the rim $O(v)$, i.e., a digital circle according to Definition 3.3. Thus, a digital model preserves the topology of a continuous space.

Now we are able to describe a method that lets us transform regions of interest produced by the image acquisition process into digital spaces with topological features of the regions.

In the first step, construct an LCL cover $\mathrm{W}$ of the region $M$ in accordance with conditions and restrictions imposed by requirements defined by the accuracy and correctness of the representation.

In the second step, build the digital model $G(W)$ of the region. Using an LCL cover guarantees that the digital model is a digital space preserving the topology of the object. 


\section{Conclusions}

This paper proposes a new discretization scheme for constructing digital models of continuous two-dimensional objects. LCL covers of a circle $C$ and Euclidean spaces $E^{1}$ and $E^{2}$ are defined, and it is shown that digital models of $C$ and $E^{1}$ are necessarily digital 1-surfaces; the digital model of $E^{2}$ is necessarily the digital 2-surface. It is shown that digital models preserve topology of their continuous counterparts, which is particularly important for many clinical and research applications.

It is shown that one can choose an LCL cover with a required resolution in any region of interest. A simple result of this consideration is that there are infinitely many non-isomorphic digital models of the plane. We describe a method that constructs digital models of areas of interest with any required resolution.

\section{References}

[1] Segonne, F. and Fischl, B. (2007) Integration of Topological Constraints in Medical Image Segmentation. Biomedical Image Analysis: Methodologies and Applications.

[2] Yan, P., Zhanga, W., Turkbey, B., Choyke, P. and Lia, X. (2013) Global Structure Constrained Local Shape Prior Estimation for Medical Image Segmentation. Computer Vision and Image Understanding, 117, 1017-1026. http://dx.doi.org/10.1016/j.cviu.2013.03.006

[3] Bai, Y., Han, X. and Prince, J. (2009) Digital Topology on Adaptive Octree Grids. Journal of Mathematical Imaging and Vision, 34, 165-184. http://dx.doi.org/10.1007/s10851-009-0140-7

[4] Brass, P. (1999) On Strongly Normal Tessellations. Pattern Recognition Letters, 20, 957-960. http://dx.doi.org/10.1016/S0167-8655(99)00063-X

[5] Brimkov, V. and Barneva, R. (2005) Analytical Honeycomb Geometry for Raster and Volume Graphics. The Computer Journal, 48, 180-199. http://dx.doi.org/10.1093/comjnl/bxh075

[6] Couprie, M., Bertrand, G. and Kenmochi, Y. (2003) Discretization in 2D and 3D orders. Graphical Models, 65, 77-91. http://dx.doi.org/10.1016/S1524-0703(03)00003-1

[7] Eckhardt, U. and Latecki, L. (2003) Topologies for the Digital Spaces $\mathrm{Z}^{2}$ and $\mathrm{Z}^{3}$. Computer Vision and Image Understanding, 90, 295-312. http://dx.doi.org/10.1016/S1077-3142(03)00062-6

[8] Evako, A. (2006) Topological Properties of Closed Digital Spaces. One Method of Constructing Digital Models of Closed Continuous Surfaces by Using Covers. Computer Vision and Image Understanding, 102, 134-144. http://dx.doi.org/10.1016/j.cviu.2003.11.003

[9] Evako, A. (2011) Characterizations of Simple Points, Simple Edges and Simple Cliques in Digital Spaces. One Method of Topology-Preserving Transformations of Digital Spaces by Deleting Simple Points and Edges. Graphical Models, 73, 1-9. http://dx.doi.org/10.1016/j.gmod.2010.10.002

[10] Kong, T., Saha, P. and Rosenfeld, A. (2007) Strongly Normal Sets of Contractible Tiles in N Dimensions. Pattern Recognition, 40, 530-543. http://dx.doi.org/10.1016/j.patcog.2005.11.013

[11] Kong, T., Saha, P. and Rosenfeld, A. (2001) Strongly Normal Sets of Tiles in N Dimensions. Electronic Notes in Theoretical Computer Science, 46, 1-12.

[12] Saha, P. and Rosenfeld, A. (1998) Strongly Normal Sets of Convex Polygons or Polyhedral. Pattern Recognition Letters, 19, 1119-1124. http://dx.doi.org/10.1016/S0167-8655(98)00088-9

[13] Evako, A., Kopperman, R. and Mukhin, Y. (1996) Dimensional Properties of Graphs and Digital Spaces. Journal of Mathematical Imaging and Vision, 6, 109-119. http://dx.doi.org/10.1007/BF00119834

[14] Ivashchenko, A. (1993) Representation of Smooth Surfaces by Graphs. Transformations of Graphs Which Do Not Change the Euler Characteristic of Graphs. Discrete Mathematics, 122, 219-233.

http://dx.doi.org/10.1016/0012-365X(93)90297-7 